\title{
The Ramsey number of diamond-matchings and loose cycles in hypergraphs
}

\author{
András Gyárfás* \\ Computer and Automation Research Institute \\ Hungarian Academy of Sciences \\ Budapest, P.O. Box 63 \\ Budapest, Hungary, H-1518 \\ gyarfas@sztaki.hu
}

Gábor N. Sárközy ${ }^{\dagger}$

Computer Science Department

Worcester Polytechnic Institute

Worcester, MA, USA 01609

gsarkozy@cs. wpi.edu

and

\author{
Endre Szemerédi \\ Computer Science Department \\ Rutgers University \\ New Brunswick, NJ, USA 08903 \\ szemered@cs.rutgers.edu
}

Computer and Automation Research Institute

Hungarian Academy of Sciences

Budapest, P.O. Box 63

Budapest, Hungary, H-1518

Submitted: Mar 4, 2008; Accepted: Oct 2, 2008; Published: Oct 13, 2008

Mathematics Subject Classification: 05C15, 05C55, 05C65

\begin{abstract}
The 2-color Ramsey number $R\left(\mathcal{C}_{n}^{3}, \mathcal{C}_{n}^{3}\right)$ of a 3 -uniform loose cycle $\mathcal{C}_{n}$ is asymptotic to $5 n / 4$ as has been recently proved by Haxell, Łuczak, Peng, Rödl, Ruciński, Simonovits and Skokan. Here we extend their result to the $r$-uniform case by showing that the corresponding Ramsey number is asymptotic to $\frac{(2 r-1) n}{2 r-2}$. Partly as a tool, partly as a subject of its own, we also prove that for $r \geq 2, R\left(k D_{r}, k D_{r}\right)=$ $k(2 r-1)-1$ and $R\left(k D_{r}, k D_{r}, k D_{r}\right)=2 k r-2$ where $k D_{r}$ is the hypergraph having $k$ disjoint copies of two $r$-element hyperedges intersecting in two vertices.
\end{abstract}

\footnotetext{
${ }^{*}$ Research supported in part by OTKA Grant No. K68322.

${ }^{\dagger}$ Research supported in part by the National Science Foundation under Grant No. DMS-0456401, by OTKA Grant No. K68322 and by a Janos Bolyai Research Scholarship.
} 


\section{Introduction}

The $r$-uniform loose cycle $\mathcal{C}_{n}^{r}$, is the hypergraph with vertex set $\{1,2, \ldots, m(r-1)=n\}$ and with the set of $m$ edges $e_{i}=\{1,2, \ldots, r\}+i(r-1), i=0,1, \ldots, m-1$ where we use $\bmod n$ arithmetic, and adding a number $t$ to a set $H$ means a shift, i.e. the set obtained by adding $t$ to each element of $H$. Notice that $\mathcal{C}_{n}^{r}$ has $n$ vertices and $m=\frac{n}{r-1}$ edges and for $r=2$ we get the usual definition of a cycle in graphs. Similarly, the $r$-uniform loose path (or shortly just a path) $\mathcal{P}_{n}^{r}$, is the hypergraph with vertex set $\{1,2, \ldots, m(r-1)+1=n\}$ and with the set of $m$ edges $e_{i}=\{1,2, \ldots, r\}+i(r-1), i=0,1, \ldots, m-1$. The Ramsey number $R\left(\mathcal{C}_{n}^{r}, \mathcal{C}_{n}^{r}\right)$ is the smallest integer $N$ for which there is a monochromatic $\mathcal{C}_{n}^{r}$ in every 2-coloring of the edges of the complete $r$-uniform hypergraph $K_{N}^{r}$. It was proved in [18] that $R\left(\mathcal{C}_{n}^{3}, \mathcal{C}_{n}^{3}\right)$ is asymptotic to $5 n / 4$. In this paper we extend that result by showing that for $r \geq 3, R\left(\mathcal{C}_{n}^{r}, \mathcal{C}_{n}^{r}\right)$ is asymptotic to $\frac{(2 r-1) n}{2 r-2}$. To see that this is about best, set $n=(2 r-2) k$ and consider the 2 -coloring of a complete graph with $(2 r-1) k-2=\frac{(2 r-1) n}{2 r-2}-2$ vertices as follows (it is a straightforward generalization of the construction of [18]) . The vertex set is partitioned into sets $A, B$ such that $|A|=k-1,|B|=(2 r-2) k-1=n-1$ and all edges within $B$ are red, the others are blue. The largest red loose cycle must be inside $B$ so it has at most $n-1$ vertices. Since all edges of a loose cycle with $m$ edges can not be met with less than $m / 2$ vertices, there is no blue loose cycle with more than $2(|A|-1)$ edges, i.e. with more than $2(r-1)(k-1)<n-1$ vertices.

In the proof we follow the argument of [18]. It uses an important tool established by Łuczak in [22] that has been successfully applied in recent results [8], [14], [15], [16], [17]. Vaguely, the method reduces the problem of finding the Ramsey number of a path or a cycle to finding the Ramsey number of a connected matching. An additional - usually technical - difficulty is that the coloring is not on the edges of a complete hypergraph but on an almost complete one, where $\epsilon\left(\begin{array}{l}n \\ r\end{array}\right)$ edges may be missing.

In order to state our main results we need a few more definitions. Let $\mathcal{H}$ be an $r$ uniform hypergraph. The shadow graph of $\mathcal{H}$ is defined as the graph $\Gamma(\mathcal{H})$ on the same vertex set, where two vertices are adjacent if they are covered by at least one edge of $\mathcal{H}$. A hypergraph is called connected if its shadow graph is connected (and its components are defined similarly). A coloring of the edges of an $r$-uniform hypergraph $\mathcal{H}, r \geq 2$, induces a multicoloring on the edges of the shadow graph $\Gamma(\mathcal{H})$ in a natural way; every edge $e$ of $\Gamma(\mathcal{H})$ receives the color of all hyperedges containing $e$. A subgraph of $\Gamma(\mathcal{H})$ is monochromatic if the color sets of its edges have a nonempty intersection.

The key element in [18] was to search for a monochromatic connected structure with many diamonds, where the diamond $D_{3}$ is two triples intersecting in two vertices. More precisely, it was proved that in any 2-coloring of the edges of an almost complete 3uniform hypergraph with $n$ vertices, there is a color, say red, such that there are vertex disjoint red diamonds covering approximately $\frac{4 n}{5}$ vertices and all of them are in the same component of the hypergraph determined by the red edges. In this paper we extend this result for the $r$-uniform diamond $D_{r}$, defined as two $r$-element edges intersecting in two vertices. (In fact, one may consider also $D_{2}$ as an edge of a graph.) The two vertices are called the central vertices of the diamond. A diamond matching is the union of vertex 
disjoint diamonds. A diamond matching is connected if all of its vertices are in the same component of the hypergraph.

Our main result is the following.

Theorem 1. Suppose that $r$ is fixed and the edges of an almost complete $r$-uniform hypergraph $\mathcal{H}$ with $n$ vertices are 2 -colored. Then there is a monochromatic connected diamond matching $k D_{r}$ such that $\left|V\left(k D_{r}\right)\right| \sim \frac{(2 r-2) n}{2 r-1}$.

Here by $\left|V\left(k D_{r}\right)\right| \sim \frac{(2 r-2) n}{2 r-1}$ we mean that $\left|V\left(k D_{r}\right)\right| \rightarrow \frac{(2 r-2) n}{2 r-1}$ as $\varepsilon \rightarrow 0$, where $\varepsilon$ is an upper bound on the fraction of the missing edges from the almost complete hypergraph.

The method of [18] can be used to derive from Theorem 1 the following.

Theorem 2. $R\left(\mathcal{C}_{n}^{r}, \mathcal{C}_{n}^{r}\right) \sim \frac{(2 r-1) n}{2 r-2}$. More precisely for all $\eta>0$ there exists $n_{0}=n_{0}(\eta)$ such that every 2-coloring of $K_{N}^{(r)}$ where $N=(1+\eta)(2 r-1) n /(2 r-2)$ contains a monochromatic copy of $\mathcal{C}_{n}^{r}$.

Partly as a tool, partly as a subject interesting in its own, we determine exactly the 2- and 3-color Ramsey numbers of a diamond-matching: $R\left(k D_{r}, k D_{r}\right)=k(2 r-1)-1$ (Theorem 4), $R\left(k D_{r}, k D_{r}, k D_{r}\right)=2 k r-2$ (Theorem 5$)$.

\subsection{Ramsey numbers for multiple copies}

If $H_{0}$ is a fixed $r$-uniform hypergraph, a multiple copy of $H_{0}$ is meant to be the hypergraph $k H_{0}$, the union of $k$ vertex disjoint copies of $H_{0}$. When $H_{0}$ is a single edge $E_{r}$, a multiple copy is usually called a matching. The Ramsey number of multiple copies of graphs has been thoroughly studied, the first such results were perhaps [4] and [6] - both in 1975 . The Ramsey number of a hypergraph matching is known exactly. The most general case is due to Alon, Frankl and Lovász (1986, [2]):

Theorem 3. Assume that $N=k r+(t-1)(k-1)$ and the edges of the complete $r$-uniform hypergraph $K_{N}^{r}$ are colored with $t$ colors. Then there is a monochromatic matching of size $k$.

One can easily see that Theorem 3 is sharp. Partition a set $S$ of $N-1$ elements into $t$ parts, $A_{1}, A_{2}, \ldots, A_{t}$ so that $\left|A_{i}\right|=k-1$ for $1 \leq i<t$. For $T \subset S,|T|=r$, color $T$ with the smallest $i$ such that $T \cap A_{i} \neq \emptyset$. Therefore - using the notation of Ramsey theory - it follows that

$$
R_{t}\left(k E_{r}\right)=R\left(k E_{r}, k E_{r}, \ldots, k E_{r}\right)=k r+(t-1)(k-1),
$$

where the dots stand for $t$ arguments. It is worth noting that Theorem 3 was conjectured by Erdös in 1973, [7] (rediscovered in [13]). Its special cases include earlier results: $r=2$ (1975, Cockayne - Lorimer, [6]), $k=2$ (this is Kneser's conjecture proved in 1978 by Lovász [21], see also Bárány [3], Green [12]) and $t=2$ (Alon and Frankl [1] and Gyárfás [13]).

Next we state and prove the Ramsey-type form of our main result, it determines the exact value of the Ramsey number of a diamond-matching. 
Theorem 4. For every $k \geq 1, r \geq 2, R\left(k D_{r}, k D_{r}\right)=k(2 r-1)-1$.

Proof. To see that the stated value is a lower bound, consider a coloring of the edges of $K_{k(2 r-1)-2}^{r}$ where all edges intersecting a fixed $(k-1)$-element subset are red and all other edges are blue.

To see that $m=k(2 r-1)-1$ is an upper bound for $R\left(k D_{r}, k D_{r}\right)$, consider a 2-coloring $c$ of $E\left(K_{m}^{r}\right)$. For every set $T \subset V\left(K_{m}^{r}\right)$ with $|T|=2 r-2$ consider the 2-coloring $c^{*}$ on the $(r-2)$-element subsets of $T$ by coloring $S \subset T,|S|=r-2$, with $c(T \backslash S)$. By Theorem $3, R\left(2 E_{r-2}, 2 E_{r-2}\right)=2(r-2)+1=2 r-3$, so there are two disjoint sets colored with the same color under $c^{*}$ and this implies that there is a monochromatic $D_{r} \subset T$ under $c$. The color of this monochromatic $D_{r}$ can be used to color $T$. Applying Theorem 3 again to this coloring, $R\left(k E_{2 r-2}, k E_{2 r-2}\right)=k(2 r-2)+k-1=k(2 r-1)-1$, so we get that there is a monochromatic $k$-matching and this gives a monochromatic $k D_{r}$, finishing the proof.

In fact, the proof method of Theorem 4 can be copied to determine the 3 -colored Ramsey number of the diamond-matching as well.

Theorem 5. For every $k \geq 1, r \geq 2, R\left(k D_{r}, k D_{r}, k D_{r}\right)=2 k r-2$.

Proof. To see that the claimed value is a lower bound, partition a $(2 k r-3)$-element set $V$ into $A_{1}, A_{2}, A_{3}$ with $\left|A_{1}\right|=\left|A_{2}\right|=k-1,\left|A_{3}\right|=k(2 r-2)-1$. Let $S \subset V,|S|=r$, and color $S$ with the minimum $i$ for which $S \cap A_{i} \neq \emptyset$.

To prove the upper bound, let $c$ be a 3-coloring of the edges of $K_{m}^{r}$ with $m=2 k r-2$.

For every set $T \subset V\left(K_{m}^{r}\right)$ with $|T|=2 r-2$ consider the 3-coloring $c^{*}$ on the $(r-2)$ element subsets of $T$ by coloring $S \subset T,|S|=r-2$, with $c(T \backslash S)$. By Theorem 3, $R\left(2 E_{r-2}, 2 E_{r-2}, 2 E_{r-2}\right)=2(r-2)+2=2 r-2$ so there are two disjoint sets colored with the same color under $c^{*}$. This implies that there is a monochromatic $D_{r} \subset T$ under $c$. The color of this monochromatic $D_{r}$ can be used to color $T$. Applying Theorem 3 again to this coloring, $R\left(k E_{2 r-2}, k E_{2 r-2}, k E_{2 r-2}\right)=k(2 r-2)+2(k-1)=2 k r-2$, so we get that there is a monochromatic $k$-matching and this gives a monochromatic $k D_{r}$, finishing the proof.

For our purposes we need a proof of Theorem 4 that carries over to almost complete hypergraphs. We use a compression principle that occurred first perhaps in [6] and in [4]. For example, a red and a blue triangle with a common vertex - called a bow tie in [11] drives the inductive argument of [4] to prove that $R\left(k K_{3}, k K_{3}\right)=5 k$ (for $k \geq 2$ ). Similar compression - a red and a blue $E_{r}$ intersecting in $r-1$ elements - makes the proof of Theorem 3 easy when $t=2$ (however, it seems that for $t>2$ the Borsuk - Ulam theorem is essential). In fact, the first author suggested the case $t=2, k=r$ as a problem for the 2007 USA Mathematical Olympiad (Problem 3 on the first day). For our case, the diamond matching, the compressed structure is a red and a blue diamond within $2 r-1$ vertices. We note here that for $r=3$ this structure played a role also in [18], (it was called a diadem there). 


\subsection{Almost complete hypergraphs, selection lemma}

Throughout this section $r \geq 2$ is a fixed integer, $0<\epsilon<1$ is arbitrarily small but fixed, $n$ approaches infinity (thus arbitrarily large). Greek letters $\delta, \rho$, etc. will be used to denote numbers that tend to zero when $\epsilon$ tends to zero ( $r$ is fixed). Hypergraph $\mathcal{H}$ is a $(1-\epsilon)$ complete $r$-uniform hypergraph on $n$ vertices, i.e. is obtained from $K_{n}^{(r)}$ by deleting at most $\epsilon\left(\begin{array}{l}n \\ r\end{array}\right)$ edges. For easier computation we shall assume that $|E(\mathcal{H})| \geq(1-\epsilon) n^{r} / r !$.

Different technical lemmas have been used earlier to handle almost complete graphs and 3-uniform hypergraphs (see [15], [18]). Here we use the concept of $\delta$-bounded selection, a tool introduced and used in [14] and in [17]. It is convenient for almost complete hypergraphs when one needs to show that there exists at least one edge at a prescribed spot or there are many edges where they need to be.

For $0<\delta<1$ fixed, we say that a sequence $L \subset V(\mathcal{H})$ of $k$ distinct vertices is obtained by a $\delta$-bounded selection (with respect to forbidden subsets of vertices) if its elements are chosen in $k$ consecutive steps so that in each step every vertex can be included as the next vertex apart from a forbidden set of at most $\delta n$ vertices. It is allowed - and that is typical in the applications - that a forbidden set for the next step depends on the sequence of previous vertices. For simplicity, sometimes we will call shortly the sequence itself a $\delta$-bounded selection. Observe that a $\delta$-bounded selection $L$ is also a $\delta^{\prime}$-bounded selection for any $\delta^{\prime}>\delta$.

In the subsequent applications when specifying a $\delta$-bounded selection of $k$ vertices in an $(1-\epsilon)$-dense hypergraph, we would like to guarantee that for every subset $S$ of the selected vertices such that $0 \leq|S| \leq r$, at least $(1-\rho) n^{r-|S|} /(r-|S|)$ ! edges of $\mathcal{H}$ contain $S$ (where $\rho$ tends to zero with $\epsilon, r, k$ are fixed). Observe that for $k=0$ we need that $\mathcal{H}$ has at least $(1-\rho) n^{r} / r$ ! edges, which is obvious with $\rho=\epsilon$. For larger $k$ our argument will be based on the following recurrence lemma (from [14]).

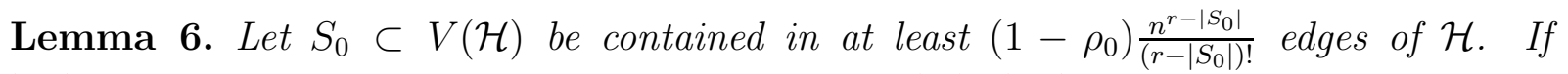
$\left|S_{0}\right|<r$ and $\rho=\sqrt{\rho_{0}}$, then there exists $F_{0} \subset V(\mathcal{H}),\left|F_{0}\right| \leq \rho n$, such that for every $x \in V(\mathcal{H}) \backslash\left(S_{0} \cup F_{0}\right)$ at least $(1-\rho) \frac{n^{r-|S|}}{(r-|S|) !}$ edges of $\mathcal{H}$ contain $S=S_{0} \cup\{x\}$.

Proof. Let $\left|S_{0}\right|=i<r$. By the assumption, there are $\beta \leq \rho_{0} n^{r-i} /(r-i)$ ! distinct $(r-i)$ element "bad" subsets $B \subseteq V(\mathcal{H}) \backslash S_{0}$ with $S_{0} \cup B \notin E(\mathcal{H})$. Let $F_{0} \subseteq V(\mathcal{H}) \backslash S_{0}$ be the set of all vertices contained in more than $\rho n^{r-i-1} /(r-i-1)$ ! distinct $(r-i)$-element bad sets. We clearly have $\beta \geq\left|F_{0}\right| \rho n^{r-i-1} /(r-i)$ !.

By comparing these two bounds on $\beta$, we obtain that $\left|F_{0}\right| \leq \frac{\rho_{0}}{\rho} n=\rho n$ and the lemma follows.

We shall use Lemma 6 to prove the following selection Lemma (its special case $k=r$ is from [14]).

Lemma 7. Assume that $\mathcal{H}$ is a $(1-\epsilon)$-complete $r$-uniform hypergraph $(r \geq 2), k$ is a positive integer, $\rho=\epsilon^{2^{-r}}, \delta=2^{k} \rho<1$. There exist forbidden sets such that for every $\delta$ bounded selection $L \subset V(\mathcal{H})$ of $k$ vertices (with respect to the forbidden sets), the following

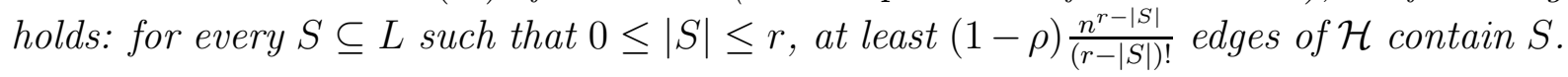


Proof. We iterate Lemma 6 as we select $x_{1}, x_{2}, \ldots, x_{k}$ in $k$ steps, in each step we consider all subsets of size less than $r$ to extend with a new vertex. At step $i$ we ensure that for every $\delta_{i}$-bounded selection $L$ of $i$ vertices the following holds: for every $S \subseteq L$ such that $0 \leq|S| \leq r$, at least $\left(1-\epsilon^{2^{-|S|}}\right) \frac{n^{r-|S|}}{(r-|S|) !}$ edges of $\mathcal{H}$ contain $S$. For $i=0, \delta_{0}=\epsilon$ obviously works. Assume this is true with $\delta_{i}$ for step $i, 0 \leq i<k$. At step $i+1$ to ensure that $x_{i+1}$ can be selected, we use Lemma 6 for all $S_{0} \subseteq\left\{x_{1}, \ldots, x_{i}\right\}$ such that $\left|S_{0}\right|<r$. By Lemma 6 , for each $j$-element $S_{0}$ there exists a forbidden set $F_{0}$ for $x_{i+1}$ with $\left|F_{0}\right| \leq \epsilon^{2^{-(j+1)}} n$ such that $S=S_{0} \cup\left\{x_{i+1}\right\}$ will be in at least $\left(1-\epsilon^{2^{-|S|}}\right) \frac{n^{r-|S|}}{(r-|S|) !}$ edges of $\mathcal{H}$. There are $\sum_{j<r}\left(\begin{array}{l}i \\ j\end{array}\right)<2^{i}$ choices for $S_{0}$ and each $j$-element $S_{0}$ forbids $\epsilon^{2^{-(j+1)}} n$ choices of $x_{i+1}$. Thus altogether the set of forbidden vertices for $x_{i+1}$ is less than $2^{i} \epsilon^{2^{-r}} n$, so $\delta_{i+1}=2^{i} \epsilon^{2^{-r}}$ is a good choice for step $i+1$. On the other hand, $\rho=\epsilon^{2^{-r}}$ is a good choice for every step since we iterate the square root operation of Lemma 6 at most $r$ times (to extend sets of size less than $r$ ).

Since

$$
\delta_{i+1}=2^{i} \epsilon^{2^{-r}} \leq 2^{k} \epsilon^{2^{-r}}=2^{k} \rho=\delta,
$$

the statement of the lemma holds with $\delta=2^{k} \epsilon^{2^{-r}}=2^{k} \rho$.

The case $|S|=r$ in Lemma 7 gives that every $r$-element set of the selected $k$ vertices is in at least $1-\rho>0$ edges of $\mathcal{H}$, thus we have the following.

Corollary 8. If $k \geq r$ then every $\delta$-bounded selection of $k$ vertices with respect to the forbidden sets ensured by Lemma 7 spans a complete $r$-uniform subhypergraph of $\mathcal{H}$.

The key in our proof of Theorem 1 is a compression lemma. We use $\mathcal{T}$ and $N$ in its formulation instead of $\mathcal{H}$ and $n$ to avoid misunderstanding when we apply it to subhypergraphs of $\mathcal{H}$. Let $\mathcal{T}$ be a $(1-\epsilon)$-complete $r$ uniform hypergraph with $N$ vertices. Assume that $x_{1}, x_{2}$ are the first two vertices of some $\delta$-bounded selection process on $\mathcal{T}$ - with $\delta=2^{k} \epsilon^{2^{-r}}$ as in Lemma 7 . Moreover, let $\mathcal{T}^{*}$ be the $(r-2)$-uniform hypergraph induced on $Z=V(T) \backslash\left\{x_{1}, x_{2}\right\}$ by $\mathcal{T}$ together with the induced 2-coloring $c\left(x_{1}, x_{2}\right)$. Notice that $\mathcal{T}^{*}$ is an $\left(1-\epsilon^{*}\right)$-complete $(r-2)$-uniform hypergraph with parameter $\epsilon^{*}=\rho=\epsilon^{2^{-r}}$. Using $\epsilon^{*}$ in the role of $\epsilon$, we can define $\delta^{*}, \rho^{*}$ as defined in Lemma $7\left(\rho^{*}=\left(\epsilon^{*}\right)^{2^{-r}}, \delta^{*}=2^{k} \rho^{*}\right)$.

Lemma 9. Assume that $\mathcal{T}$ is a 2-colored $(1-\epsilon)$-complete $r$-uniform hypergraph on $N$ vertices. Suppose that the pair $x_{1}, x_{2} \in V(\mathcal{T})$ is in at least $\mu\left(\begin{array}{c}N \\ r-2\end{array}\right)$ edges in both colors, where $\mu=1-\left(1-\rho^{*}\right)^{r-2}$. Then one can find a diamond in both colors within $2 r-1$ vertices.

Proof. Set $k=4(r-2)$ and apply Lemma 7 to $\mathcal{T}$ and $\mathcal{T}^{*}$ simultaneously in the following way. Starting with $x_{1}, x_{2}$, continue the sequence $x_{1}, x_{2}, y_{1}, y_{2}, \ldots, y_{k}$ of vertices of $\mathcal{T}$ so that at each step $y_{i}$ is selected outside the union of the forbidden set for $\mathcal{T}$ and the forbidden set of $\mathcal{T}^{*}$. Then at each step we have a forbidden set of size at most $\left(\delta+\delta^{*}\right) N \leq 2 \delta^{*} \mathrm{~N}$, thus we can define selections $x_{1}, x_{2}, y_{1}, y_{2}, \ldots, y_{k}$ that is $2 \delta^{*}$-bounded on $\mathcal{T}$ and $y_{1}, y_{2}, \ldots, y_{k}$ is $2 \delta^{*}$-bounded on $\mathcal{T}^{*}$. This ensures, by Corollary 8, that the $r$-uniform subhypergraph of $\mathcal{T}$ spanned by $x_{1}, x_{2}, y_{1}, y_{2}, \ldots, y_{k}$ and the $(r-2)$-uniform subhypergraph of $\mathcal{T}^{*}$ spanned by $y_{1}, y_{2}, \ldots, y_{k}$ are complete subhypergraphs. 
Fix an edge $e \in \mathcal{T}^{*}$ with vertex set $\left\{y_{1}, \ldots, y_{r-2}\right\}$, say $e$ is red under $c\left(x_{1}, x_{2}\right)$. Consider the subhypergraph $\mathcal{F}$ of $\mathcal{T}^{*}$ with edges that can be obtained as the next $r-2$ vertices, $y_{r-1}, \ldots y_{2 r-4}$ in the selection. The choice of $\mu$ and the lower bound on the number of blue edges ensures that at least one edge $f \in \mathcal{F}$ is blue (under $c\left(x_{1}, x_{2}\right)$ ):

$$
|\mathcal{F}|>\frac{\left(1-2 \rho^{*}\right)^{r-2} N^{r-2}}{(r-2) !}=\frac{(1-\mu) N^{r-2}}{(r-2) !}>(1-\mu)\left(\begin{array}{c}
N \\
r-2
\end{array}\right) \geq\left|E\left(\mathcal{T}_{R}^{*}\right)\right|
$$

where $\mathcal{T}_{R}^{*}$ is the set of hyperedges of $\mathcal{T}$ colored with red by $c\left(x_{1}, x_{2}\right)$. Consider the complete $r-2$-uniform hypergraph $\mathcal{F} \subset \mathcal{T}^{*}$ spanned by the vertex set of $e \cup f$. Among all pairs of edges of $\mathcal{F}$ with distinct colors (there are pairs like that: $e, f$ ) select a pair $R_{1}, B_{1}$ with the largest intersection. Clearly, $\left|R_{1} \cap B_{1}\right|=r-3$.

Repeat the previous procedure by fixing an edge with vertices $y_{2 r-3}, \ldots y_{3 r-2}$ in $\mathcal{T}^{*}$ then find an edge of the other color. By taking a pair with the largest intersection again, we have another red-blue pair of edges $R_{2}, B_{2}$ such that $\left|R_{2} \cap B_{2}\right|=r-3$. Notice that $R_{1} \cup B_{1}$ and $R_{2} \cup B_{2}$ are vertex disjoint. Define $r_{1}=R_{1} \backslash B_{1}, r_{2}=R_{2} \backslash B_{2}, b_{1}=B_{1} \backslash R_{1}$, $b_{2}=B_{2} \backslash R_{2}$.

Notice that the (complete) subhypergraph of $\mathcal{T}$ spanned by $\left\{x_{1}, x_{2}\right\} \cup R_{1} \cup R_{2} \cup B_{1} \cup B_{2}$ has $2 r$ vertices and contains $D_{r}$ in both colors. To finish the proof, we need to find a vertex whose deletion keeps a copy of $D_{r}$ in both colors.

Consider the $r$-element set $U_{1}$ that is the union of $B_{2}$, one vertex of $R_{1} \cap B_{1}$ and the vertex $r_{1}$. (In case of $r=3, R_{1} \cap B_{1}$ is empty - then we can select $x_{1}$ as the third vertex and $r_{2}$ or $b_{1}$ can be removed, the argument ends here.) If $U_{1}$ is red (under $c$ ) then the vertex $r_{2}$ can be removed and we get both red and blue diamonds within $2 r-1$ vertices. Thus we may assume that $U_{1}$ is blue. Similar argument gives that $U_{2}$, defined as the union of $R_{1}$, one vertex of $R_{2} \cap B_{2}$ and the vertex $b_{2}$ is red. Likewise, $U_{3}$ defined as the union of $B_{1}$, one vertex of $R_{2} \cap B_{2}$ and the vertex $r_{2}$ is blue, finally $U_{4}$, defined as the union of $R_{2}$, one vertex of $R_{1} \cap B_{1}$ and the vertex $b_{1}$ is red. Now $U_{1} \cup U_{3}$ and $U_{2} \cup U_{4}$ are the required diamonds (in fact they are within $2 r-2$ vertices).

\section{Proof of Theorem 1}

Assume that $\mathcal{H}$ is $(1-\epsilon)$-complete. We start by fixing the upper bound of $\epsilon$ under which our argument works. Initially we select $\delta$ to satisfy Lemma 7 , i.e. $\delta \leq 2^{k} \rho=2^{k} \epsilon 2^{-r}$ but we also need Lemma 9 to make $2 \delta^{*}$-bounded selections of $k=4(r-2)$ vertices in $\left(1-\epsilon^{*}\right)$-complete hypergraphs. Thus - with a bit generously - we bound $\epsilon$ (in terms of our fixed $r$ ) by requiring

$$
2 \delta^{*}=2^{k+1} \rho^{*}=2^{k+1} \epsilon^{* 2^{-r}}=2^{4 r+1} \epsilon^{4^{-r}}<1 .
$$

To prove Theorem 1, consider a 2-coloring $c$ of an $(1-\epsilon)$-complete $r$-uniform hypergraph $\mathcal{H}$ with $\epsilon$ bounded by (1). Let $\mathcal{H}_{R}, \mathcal{H}_{B}$ denote the the hypergraphs determined by the red and blue edges of $\mathcal{H}$. We start with some observations about the monochromatic components of $\mathcal{H}$ which leads to distinguishing some cases (A, B1 and B2). We apply the following proposition from [14]. 
Proposition 10. Assume $\mathcal{H}$ is an arbitrary hypergraph and $0<\lambda<1 / 3$. It is either possible to delete at most $\lambda n$ vertices from $\mathcal{H}$ so that the remaining hypergraph $\mathcal{H}^{\prime}$ is connected or the connected components of $\mathcal{H}$ can be partitioned into two groups so that each group contains more than $\lambda n$ vertices.

Proof. Mark the connected components of $\mathcal{H}$ until the union of them has at most $\lambda n$ vertices. If one unmarked component remains, let it be $\mathcal{H}^{\prime}$. Otherwise, we form two groups from the unmarked components. The larger group has order at least $(n-\lambda n) / 2>\lambda n$, and the smaller one together with the marked components have a union containing more than $\lambda n$ vertices as well.

We start by applying Proposition 10 to $\mathcal{H}_{R}$ and to $\mathcal{H}_{B}$ with $\lambda$ that tends to zero with $\epsilon$.

If the first possibility holds to one of them, say to $\mathcal{H}_{R}$, we find a subhypergraph $\mathcal{H}_{1}$ with at least $(1-\lambda) n$ vertices that is connected in red. Now apply Proposition 10 again to the hypergraph determined by the blue edges of $\mathcal{H}_{1}$. If the first possibility holds then we have a subhypergraph $\mathcal{H}_{2}$ of $\mathcal{H}_{1}$ with at least $(1-2 \lambda) n$ vertices that is connected in blue and also part of the connected red hypergraph $\mathcal{H}_{1}$. Since we loose at most $2 \lambda n=o(n)$ vertices, for convenience, we still use the notation $\mathcal{H}$ for $\mathcal{H}_{i}$ and consider this as case A. To comply with the notation of cases B1, B2 below, set $Y=V$ in case A.

Assume that the first possibility does not hold for at least one of the steps above, this is case B. We may assume that it does not hold in the first step. We look at two subcases. Apply again Proposition 10 to $\mathcal{H}_{R}$ but with $\lambda=\frac{1}{2 r-1}$. Note that $s<\frac{1}{3}$ since $r \geq 3$.

If the first possibility holds, the vertex set of $\mathcal{H}$ is partitioned into $X$ and $Y$ such that $|X|<\frac{n}{2 r-1}$ and $\mathcal{H}_{R}$ spans a connected red hypergraph on $Y$, this is subcase B1.

If the second possibility holds, the vertex set of $\mathcal{H}$ can be partitioned into $X$ and $Y$ such that $\frac{n}{2 r-1} \leq|X| \leq|Y|$, this is subcase $\mathrm{B} 2$.

Notice that (in both subcases) all edges of $\mathcal{H}$ meeting both $X$ and $Y$ are blue.

Continuing the proof of Theorem 1, we try to cover as many vertices of $Y$ as we can with pairwise disjoint sets $S_{i}, i=1,2, \ldots m$ that contain diamonds of both colors and $\left|S_{i}\right|=2 r-1$. Set $S=\cup_{i=1}^{m} S_{i}, T=Y \backslash S$. The hypergraphs induced by $\mathcal{H}$ on $S, T$ are denoted by $\mathcal{S}, \mathcal{T}$. Since Lemma 9 does not give a new $S_{i} \subset T$, for every pair $x_{1}, x_{2} \in T$ there is a color such that there are more than $(1-\mu)\left(\begin{array}{c}|T| \\ r-2\end{array}\right)$ edges in that color in the coloring $c\left(x_{1}, x_{2}\right)$. Assign that color to the pair $x_{1}, x_{2}$, to get a 2-coloring $C$ on the graph $G$ whose edges are the pairs available as the first two vertices on a $\delta$-bounded selection on $\mathcal{T}$. Notice that $G$ is an $(1-2 \delta)$-complete graph.

We claim that $\mathcal{T}$ has an almost perfect monochromatic diamond matching $\mathcal{M}$ (i.e. $V(\mathcal{T})$ can be partitioned into vertex disjoint diamonds all of the same color, apart from $o(n)$ vertices.) First we show that almost all edges of $G$ are colored with the same color (under $C$ ). Indeed, otherwise - using that $G$ is almost complete - we could easily find a red edge $u v$ and a blue edge $v w$ of $G$. Define a coloring $c^{*}$ by restricting the colorings $c(u, v), c(v, w)$ to the hypergraph $\mathcal{T}^{*}$ whose edges are the $(r-2)$-element subsets $e \subset T$ for which $e \cup\{u, v\}$ and $e \cup\{v, w\}$ are both in $\mathcal{H}$. Observe that $c^{*}$ colors every edge of an $\left(1-2 \mu-2 \epsilon^{*}\right)$-complete $(r-2)$-uniform hypergraph with both red and blue colors. 
Then one can make a $\delta$-bounded selection $u, v, w, y_{1}, \ldots y_{2 r-4}$ such that $y_{1}, \ldots y_{2 r-4}$ spans a $K_{r-2}^{2(r-2)}$ with all edges colored in both colors. In particular, we have a red and a blue $D_{r}$ within $2 r-1$ vertices of $T$, contradicting the choice of $m$. Thus almost all edges of $G$ have the same color, implying that almost all edges of $\mathcal{T}$ have the same color, i.e. $\mathcal{T}$ is an almost complete hypergraph in one of the two colors, so certainly has an almost perfect monochromatic diamond matching $\mathcal{M}$ as required.

In case A both colors define a connected hypergraph so the diamonds in the color of $\mathcal{M}$ together with the diamonds of the appropriate color from the $S_{i}$-s provide the monochromatic connected diamond matching, covering approximately a portion of $\frac{2 r-2}{2 r-1}$ of the vertex set of $\mathcal{H}$.

In case B2 it easy to cover the required portion of vertices by blue diamonds since all edges meeting both $X$ and $Y$ are blue and $\frac{n}{2 r-1} \leq|X| \leq|Y|$ (connectivity of the blue hypergraph is obvious). In fact, one can cover approximately $\frac{(2 r-2) n}{2 r-1}$ vertices with vertex disjoint blue diamonds using only diamonds of type $(1,2 r-3)$ and $(2 r-3,1)$ where type $(a, b)$ means a diamond intersecting $X, Y$ in $a$ and $b$ vertices, respectively, with its center vertices in $X, Y$. The reason is that flipping one blue diamond in a diamond matching from type $(1,2 r-3)$ to type $(2 r-3,1)$ changes the cover ratio of $Y$ and $X$ by at most a quantity that tends to zero if $n$ tends to infinity ( $r$ is fixed). The details are left to the reader. This argument extends to case B1 as well, if $m \geq \frac{n}{2 r-1}-|X|$ : in addition to the blue diamonds meeting both $X$ and $Y$ we can use the blue diamonds of $S_{i}$. Thus we may assume that $m<\frac{n}{2 r-1}-|X|$.

If $\mathcal{M}$ is red then the diamonds of $\mathcal{M}$ together with the red diamonds of the $S_{i}$-s cover all but $m+|X|<\frac{n}{2 r-1}-|X|+|X|=\frac{n}{2 r-1}$ vertices, finishing the proof. If $\mathcal{M}$ is blue we can do the same in blue - here we gain since all diamonds meeting $X$ and vertices uncovered by the blue diamonds of $S_{i}$ are giving extra to the covered area. This finishes the proof of Theorem 1 .

\section{From connected diamond matchings to loose cycles}

For the sake of completeness here we sketch how the method of [18] with minor modifications (that are needed since the uniformity is $r$ instead of 3) can be used to transform our asymptotic result on monochromatic connected diamond matchings (Theorem 1) to our asymptotic result on monochromatic loose cycles (Theorem 2). The missing details can be found in [18].

The main tool is the hypergraph version of the Regularity Lemma of Szemerédi [24]. We shall assume throughout the rest of the paper that $n$ is sufficiently large and $r$ is fixed.

There are several generalizations of the Regularity Lemma for hypergraphs due to various authors ([5], [9], for an extensive survey see [20], new developments are in [10], [23] and [25]). Following [18], the simplest one, due to Chung [5] can be used. To state it, one needs to define the notion of $\varepsilon$-regularity. Let $\varepsilon>0$ and let $V_{1}, V_{2}, \ldots, V_{r}$ be disjoint vertex sets of order $m$, and let $\mathcal{H}$ be an $r$-uniform hypergraph such that every edge of $\mathcal{H}$ contains exactly one vertex from each $V_{i}$ for $i=1,2, \ldots, r$. The density of $\mathcal{H}$ is 
$d_{\mathcal{H}}=\frac{|E(\mathcal{H})|}{m^{r}}$. The $r$-tuple $\left(V_{1}, V_{2}, \ldots, V_{r}\right)$ is called an $(\varepsilon, \mathcal{H})$-regular $r$-tuple of density $d_{\mathcal{H}}$ if for every choice of $X_{i} \subset V_{i},\left|X_{i}\right|>\varepsilon\left|V_{i}\right|, i=1,2, \ldots, r$ we have

$$
\left|\frac{\left|E\left(\mathcal{H}\left[X_{1}, \ldots, X_{r}\right]\right)\right|}{\left|X_{1}\right| \ldots\left|X_{r}\right|}-d_{\mathcal{H}}\right|<\varepsilon
$$

Here we denote by $\mathcal{H}\left[X_{1}, \ldots, X_{r}\right]$ the subhypergraph of $\mathcal{H}$ induced by the vertex set $X_{1} \cup \ldots \cup X_{r}$. Similarly as in [18] for $r=3$, we need a 2-color version of the Hypergraph Regularity Lemma from [5] for general $r$.

Lemma 11 (2-color Weak Hypergraph Regularity Lemma). For every positive $\varepsilon$ and positive integers $t, r$ there are positive integers $M$ and $n_{0}$ such that for $n \geq n_{0}$ the following holds. For all r-uniform hypergraphs $\mathcal{H}_{1}, \mathcal{H}_{2}$ with $V\left(\mathcal{H}_{1}\right)=V\left(\mathcal{H}_{2}\right),|V|=n$, there is a partition of $V$ into $l+1$ classes (clusters)

$$
V=V_{0}+V_{1}+V_{2}+\ldots+V_{l}
$$

such that

- $t \leq l \leq M$

- $\left|V_{1}\right|=\left|V_{2}\right|=\ldots=\left|V_{l}\right|$

- $\left|V_{0}\right|<\varepsilon n$

- $\quad$ apart from at most $\varepsilon\left(\begin{array}{l}l \\ r\end{array}\right)$ exceptional $r$-tuples, the $r$-tuples $\left\{V_{i_{1}}, V_{i_{2}}, \ldots, V_{i_{r}}\right\}$ are $\left(\varepsilon, \mathcal{H}_{s}\right)$-regular for $s=1,2$.

Consider a 2-edge coloring $\left(\mathcal{H}_{1}, \mathcal{H}_{2}\right)$ of the $r$-uniform complete hypergraph $K_{N}^{(r)}$, where $N=(1+\eta)(2 r-1) n /(2 r-2)$, i.e. $\mathcal{H}_{1}$ is the subhypergraph induced by the first color (say red) and $\mathcal{H}_{2}$ is the subhypergraph induced by the second color (say blue).

We apply the above 2-color Weak Hypergraph Regularity Lemma with $t=r$ and with a small enough $\varepsilon$ to obtain a partition of $V\left(K_{N}^{(r)}\right)=V=\cup_{0 \leq i \leq l} V_{i}$, where $\left|V_{i}\right|=\frac{N-\left|V_{0}\right|}{l}=$ $m, 1 \leq i \leq l$. We define the following reduced hypergraph $\mathcal{H}^{\bar{R}}$ : The vertices of $\mathcal{H}^{R}$ are $p_{1}, \ldots, p_{l}$, and we have an $r$-edge on vertices $p_{i_{1}}, p_{i_{2}}, \ldots, p_{i_{r}}$ if the $r$-tuple $\left(V_{i_{1}}, V_{i_{2}}, \ldots, V_{i_{r}}\right)$ is $\left(\varepsilon, \mathcal{H}_{s}\right)$-regular for $s=1,2$. Thus we have a one-to-one correspondence $f: p_{i} \rightarrow V_{i}$ between the vertices of $\mathcal{H}^{R}$ and the clusters of the partition. Then,

$$
\left|E\left(\mathcal{H}^{R}\right)\right| \geq(1-\varepsilon)\left(\begin{array}{l}
l \\
r
\end{array}\right),
$$

and thus $\mathcal{H}^{R}$ is a $(1-\varepsilon)$-complete $r$-uniform hypergraph on $l$ vertices. Define a 2-edge coloring $\left(\mathcal{H}_{1}^{R}, \mathcal{H}_{2}^{R}\right)$ of $\mathcal{H}^{R}$ with the majority color, i.e. the $r$-tuple $\left\{p_{i_{1}}, p_{i_{2}}, \ldots, p_{i_{r}}\right\} \in$ $E\left(\mathcal{H}_{s}^{R}\right)$ if $s$ is the more frequent color in the $r$-tuple $\left(V_{i_{1}}, V_{i_{2}}, \ldots, V_{i_{r}}\right) \in E\left(\mathcal{H}_{s}\right)$. Note then that the density of this color is $\geq 1 / 2$ in this $r$-tuple. Finally we consider the multicolored shadow graph $\Gamma\left(\mathcal{H}^{R}\right)$. The vertices are $V\left(\mathcal{H}^{R}\right)=\left\{p_{1}, \ldots, p_{l}\right\}$ and we join vertices $x$ and 
$y$ by an edge of color $s, s=1,2$ if $x$ and $y$ are contained in an edge of $\mathcal{H}^{R}$ that is colored with color $s$.

Applying Theorem 1 to the 2-colored almost complete reduced graph $\mathcal{H}^{R}$ we get a monochromatic (say red) connected diamond matching $D_{r}^{1}, \ldots, D_{r}^{k}$ with $k(2 r-2) \sim$ $\frac{(2 r-2) l}{2 r-1}$, i.e. $k \sim l /(2 r-1)$. Let $\mathcal{L}$ be the red component of $\mathcal{H}^{R}$ that contains these diamonds.

Applying the method of [18] to find the red $\mathcal{C}_{n}^{r}$ we do the following. We first trace a closed "route" in $\mathcal{L}$, that visits all the diamonds $D_{r}^{1}, \ldots, D_{r}^{k}$. Then we choose a collection of short loose paths (of length three or six where we count edges) in the red subhypergraph $\mathcal{H}_{1}$, that link together to form a short loose cycle, following the chosen route. Finally, to obtain the red loose cycle $\mathcal{C}_{n}^{r}$ we "blow-up" $k \sim l /(2 r-1)$ short paths (of length three) corresponding to diamonds by long paths (each of length $\sim 2 m$, so covering $\sim$ $(2 r-2) m \sim(2 r-2) N / l \sim(2 r-1) n / l$ vertices $)$. More precisely, for each diamond $D_{r}^{i}$ with middle clusters $V_{1}^{i}$ and $V_{2}^{i}$, we replace the short path that starts in $V_{1}^{i}$ and ends in $V_{2}^{i}$ by a long path with the same end-vertices, that uses almost all the vertices in $D_{r}^{i}$. Note that these long paths are mutually vertex disjoint since all diamonds $D_{r}^{i}$ are vertex disjoint. Therefore, to obtain our cycle, we just need to make sure that the short paths do not intersect and they do not interfere with the long paths. This will be guaranteed by the introduction of the set $B$ in the lemmas below.

This plan can be achieved via the same sequence of lemmas as in [18]. To demonstrate what kind of minor modifications are needed in these lemmas for $r$-uniform hypergraphs, we present the modified version of perhaps the most important lemma, Lemma 5.3 in [18], that shows how to find the short connecting loose paths of length three. First we need the following definition.

Let $\left(V_{i_{1}}, \ldots, V_{i_{r}}\right)$ be an $\left(\varepsilon, \mathcal{H}_{1}\right)$-regular $r$-tuple with density $d>2 \varepsilon$, and for $j=1, \ldots, r$ let $U_{i_{j}} \subset V_{i_{j}}$ be arbitrary subsets. We say that a vertex $x \in V_{i_{1}}$ is good for the $r$ tuple $\left(U_{i_{1}}, \ldots, U_{i_{r}}\right)$ if for every $j=2, \ldots, r$ there are at least $d\left|U_{i_{j}}\right| / 2$ vertices $y \in U_{i_{j}}$, such that for each such $y$, there are at least $d\left|U_{i_{2}}\right| / 2$ vertices $z_{1} \in U_{i_{2}}$, such that for each such $z_{1}$, there are at least $d\left|U_{i_{3}}\right| / 2$ vertices $z_{2} \in U_{i_{3}}$, etc. we go through the sets $U_{i_{j^{\prime}}}, j^{\prime}=2, \ldots, r, j^{\prime} \neq j$ with this process, finally there at least $d\left|U_{i_{r}}\right| / 2$ vertices $z_{r-2} \in U_{i_{r}}$, such that $\left\{x, y, z_{1} \ldots, z_{r-2}\right\} \in E\left(\mathcal{H}_{1}\right)$. Thus note that for $x \in V_{i_{1}}$, the property of being good for $\left(U_{i_{1}}, \ldots, U_{i_{r}}\right)$ is independent of the choice of $U_{i_{1}}$. The set of vertices in $V_{i_{1}} \cup \ldots \cup V_{i_{r}}$ that are good for $\left(V_{i_{1}}, \ldots, V_{i_{r}}\right)$ will simply be called good.

We modify Lemma 5.3 of [18] in the following way for $r$-uniform hypergraphs.

Lemma 12. Let $\left(V_{i_{1}}, \ldots, V_{i_{r}}\right)$ be an $\left(\varepsilon, \mathcal{H}_{1}\right)$-regular $r$-tuple with density $d>2 \varepsilon$. Then for every pair of good vertices $x \in V_{i_{1}}$ and $y \in V_{i_{2}}$, and for every set $B \subset V_{i_{1}} \cup \ldots \cup V_{i_{r}} \backslash\{x, y\}$ that contains all non-good vertices and satisfies $\left|B \cap V_{i_{j}}\right|<(d / 2-\varepsilon) m$ for $j=1, \ldots, r$, there is a path of length three in $\mathcal{H}_{1}$ joining $x$ to $y$ that is disjoint from $B$ (and hence contains only good vertices). Moreover the path can be chosen so that one vertex of degree two in the path is in $V_{i_{1}}$, and the other is in $V_{i_{2}}$.

Proof. Since $x$ is good, there exists a set $U_{x} \subset V_{i_{2}},\left|U_{x}\right| \geq d m / 2$ (using $j=2$ from the definition), such that for each $w \in U_{x}$, there are at least $d m / 2$ vertices $z_{1} \in V_{i_{3}}$, such 
that for each such $z_{1}$, there are at least $d m / 2$ vertices $z_{2} \in V_{i_{4}}$, etc., finally there are at least $d m / 2$ vertices $z_{r-2} \in V_{i_{r}}$, such that $\left\{x, w, z_{1} \ldots, z_{r-2}\right\} \in E\left(\mathcal{H}_{1}\right)$. Similarly, since $y$ is good, there exists a set $U_{y} \subset V_{i_{1}},\left|U_{y}\right| \geq d m / 2$ (using $j=1$ from the definition), such that for each $v \in U_{y}$, there are at least $d m / 2$ vertices $z_{1} \in V_{i_{3}}$, such that for each such $z_{1}$, there are at least $d m / 2$ vertices $z_{2} \in V_{i_{4}}$, etc., finally there are at least $d m / 2$ vertices $z_{r-2} \in V_{i_{r}}$, such that $\left\{y, v, z_{1} \ldots, z_{r-2}\right\} \in E\left(\mathcal{H}_{1}\right)$. Writing $b=(d / 2-\varepsilon)$, we have

$$
\left|U_{x} \backslash B\right|,\left|U_{y} \backslash B\right|>(d / 2-b) m=\varepsilon m .
$$

Therefore, since $\left(V_{i_{1}}, \ldots, V_{i_{r}}\right)$ is an $\left(\varepsilon, \mathcal{H}_{1}\right)$-regular $r$-tuple with density $d$, we know that

$$
\begin{gathered}
\left|E\left(\mathcal{H}\left[U_{x} \backslash B, U_{y} \backslash B, V_{i_{3}} \backslash B, \ldots, V_{i_{r}} \backslash B\right]\right)\right| \geq \\
\geq(d-\varepsilon)\left|U_{x} \backslash B\right|\left|U_{y} \backslash B\right| \prod_{j=3}^{r}\left|V_{i_{j}} \backslash B\right| .
\end{gathered}
$$

We may therefore choose distinct vertices $w \in U_{x} \backslash\{y\}, v \in U_{y} \backslash\{x\}, z_{1}^{1}, z_{1}^{2}, z_{1}^{3} \in V_{i_{3}}$, $z_{2}^{1}, z_{2}^{2}, z_{2}^{3} \in V_{i_{4}}, \ldots, z_{r-2}^{1}, z_{r-2}^{2}, z_{r-2}^{3} \in V_{i_{r}}$ such that

$$
\left\{x, w, z_{1}^{1}, \ldots, z_{r-2}^{1}\right\},\left\{y, v, z_{1}^{2}, \ldots, z_{r-2}^{2}\right\},\left\{v, w, z_{1}^{3}, \ldots, z_{r-2}^{3}\right\} \in E\left(\mathcal{H}_{1}\right) .
$$

This gives us the required loose path of length three joining $x$ to $y$

$$
x, z_{1}^{1}, \ldots, z_{r-2}^{1}, w, z_{1}^{3}, \ldots, z_{r-2}^{3}, v, z_{1}^{2}, \ldots, z_{r-2}^{2}, y .
$$

Lemma 12 tells us how to find the short connecting loose paths of length three in the above outline. Then we blow-up these loose paths of length three by the help of the following lemma. This lemma is again a similarly modified version of Lemma 5.5 of [18], so we omit the details.

Lemma 13. Let $V_{i_{1}}, V_{i_{2}}, V_{i_{3}}, \ldots, V_{i_{r}}, V_{i_{3}}^{\prime}, \ldots, V_{i_{r}}^{\prime}$ be $(2 r-2)$ clusters given in such a way that $\left(V_{i_{1}}, V_{i_{2}}, V_{i_{3}}, \ldots, V_{i_{r}}\right)$ and $\left(V_{i_{1}}, V_{i_{2}}, V_{i_{3}}^{\prime}, \ldots, V_{i_{r}}^{\prime}\right)$ are both $\left(\varepsilon, \mathcal{H}_{1}\right)$-regular $r$-tuples with density $d \gg \varepsilon$. Let $x \in V_{i_{1}}$ and $y \in V_{i_{2}}$ be good vertices for both $r$-tuples. Let $B \subset$ $V_{i_{1}} \cup \ldots \cup V_{i_{r}} \cup V_{i_{3}}^{\prime} \cup \ldots \cup V_{i_{r}}^{\prime} \backslash\{x, y\}$ be such that $B$ contains all non-good vertices for either $r$-tuples and satisfies $|B \cap V|<2 \varepsilon m$ for all $V \in\left\{V_{i_{1}}, V_{i_{2}}, V_{i_{3}}, \ldots, V_{i_{r}}, V_{i_{3}}^{\prime}, \ldots, V_{i_{r}}^{\prime}\right\}$. Then there is a path joining $x$ and $y$ in the union of the two $r$-tuples of length $l$ for all odd integers $l$ satisfying $d m \leq l \leq\left(1-\varepsilon^{\prime}\right) 2 m$ (for some $\varepsilon \ll \varepsilon^{\prime} \ll \eta$ ) that avoids $B$.

Note again that the set $B$ here is used to make sure that short and long paths do not intersect. The other lemmas and the proof itself can be modified similarly, details can be found in [18]. This finishes the proof of Theorem 2.

Acknowledgement. Thanks to an unknown referee whose useful remarks improved the presentation. 


\section{References}

[1] N. Alon, P. Frankl, Families in which disjoint sets have large union, in Combinatorial Mathematics; Proceedings of the Third International Conference, New York, 1985 Ann. New York Acad. Sci. 555 (1989), pp. 9-16.

[2] N. Alon, P. Frankl, L. Lovász, The chromatic number of Kneser hypergraphs, Transactions of the American Mathematical Society 298 (1986), pp. 359-370.

[3] I. Bárány, A short proof of Kneser's conjecture, J. of Comb. Theory A, 25 (1978), 325-326.

[4] S. A. Burr, P. Erdős, J. H. Spencer, Ramsey theorems for multiple copies graphs, Transactions of the American Mathematical Society 209 (1975), pp. 87-99.

[5] F. Chung, Regularity lemmas for hypergraphs and quasi-randomness, Random Structures and Algorithms 2 (1991), pp. 241-252.

[6] E. J. Cockayne, P. J. Lorimer, The Ramsey number for stripes, J. Austral. Math. Soc. 19 (1975), pp. 252-256.

[7] P. Erdős, Problems and results in combinatorial analysis, Colloq. Internat. Theor. Combin. Rome 1973, Acad. Naz. Lincei, Rome (1976), pp. 3-17.

[8] A. Figaj, T. Euczak, The Ramsey number for a triple of long even cycles, Journal of Combinatorial Theory, Ser. B, 97 (2007), pp. 584-596.

[9] P. Frankl, V. Rödl, The uniformity lemma for hypergraphs, Graphs and Combinatorics 8 (1992), pp. 309-312.

[10] W.T. Gowers, Hypergraph regularity and the multidimensional Szemerédi Theorem, Ann. of Math. 166 (2007), no. 3, pp. 897-946.

[11] R. L. Graham, B. L. Rothschild, J. H. Spencer, Ramsey Theory, John Wiley \& Sons, 1990.

[12] J. Green, A new short proof of Kneser's conjecture, American Mathematical Monthly, 109, (2002), pp. $918-920$.

[13] A. Gyárfás, On the Ramsey number of disjoint hyperedges, unpublished manuscript.

[14] A. Gyárfás, J. Lehel, G. N. Sárközy, R. H. Schelp, Monochromatic Hamiltonian Berge cycles in colored complete hypergraphs, Journal of Combinatorial Theory, Ser. B, 98 (2008), pp. 342-358.

[15] A. Gyárfás, M. Ruszinkó, G. N. Sárközy, E. Szemerédi, Three-color Ramsey numbers for paths, Combinatorica, 27 (2007), pp. 35-69.

[16] A. Gyárfás, M. Ruszinkó, G. N. Sárközy, E. Szemerédi, Tripartite Ramsey numbers for paths, Journal of Graph Theory 55 (2007), pp. 164-174.

[17] A. Gyárfás, G. N. Sárközy, The 3-color Ramsey number of a 3-uniform Berge-cycle, submitted, WPI Technical Report WPICS-CS-TR-07-12, http://www.cs.wpi.edu/Research/techreports.html 
[18] P. Haxell, T. Łuczak, Y. Peng, V. Rödl, A. Ruciński, M. Simonovits, J. Skokan, The Ramsey number for hypergraph cycles I, Journal of Combinatorial Theory, Ser. A 113 (2006), pp. 67-83.

[19] P. Haxell, T. Łuczak, Y. Peng, V. Rödl, A. Ruciński, J. Skokan, The Ramsey number for hypergraph cycles II, London School of Economics, CDAM report, 2007 - 05.

[20] J. Komlós, M. Simonovits, Szemerédi's Regularity Lemma and its applications in graph theory, in Combinatorics, Paul Erdős is Eighty (D. Miklós, V.T. Sós, and T. Szőnyi, Eds.), Bolyai Society Math. Studies, Vol.2, pp. 295-352, Budapest, 1996.

[21] L. Lovász, Kneser's conjecture, chromatic numbers and homotopy, J. Comb. Theory A, 25 (1978), pp. 319-324.

[22] T. Luczak, $R\left(C_{n}, C_{n}, C_{n}\right) \leq(4+o(1)) n$, Journal of Combinatorial Theory, Ser. $B$ 75 (1999), pp. 174-187.

[23] V. Rödl, J. Skokan, Regularity Lemma for uniform hypergraphs, Random Structures and Algorithms 25 (1) (2004), pp. 1-42.

[24] E. Szemerédi, Regular partitions of graphs, Colloques Internationaux C.N.R.S. № 260 - Problèmes Combinatoires et Théorie des Graphes, Orsay (1976), pp. 399-401.

[25] T. Tao, A variant of the hypergraph removal lemma, Journal of Combinatorial Theory, Ser. A 113 (2006), pp. 1257-1280. 